\section{Duplication: stop favouring applicant with longest list}

SIR - The issue discussed in Mounir Errami and Harold Garner's Commentary 'A tale of two citations' (Nature 451, 397-399; 2008) is a real problem, although I don't believe there are more duplicate papers than 10 years ago. This problem will only go away if grants and jobs are no longer given to those with the longest publication record.

Alternatives? Ask applicants to select their best three, five or ten papers.

Martin Fenner

Department of Haematology, Haemostaseology and Oncology, Hannover Medical School,

30625 Hannover, Germany

\section{Duplication spreads the word to a wider audience}

SIR - Self-plagiarism seems to be an endemic phenomenon in biomedical journals, according to a recent Nature News discussion (http://tinyurl.com/2fcnfg) of the Commentary 'A tale of two citations' (Nature 451, 397-399; 2008). Classical examples include redundant publication, duplicate publication and text recycling. But is selfplagiarism really a bad thing?

We should try to disseminate scientific knowledge to the largest audience possible in order to help people solve their problems. This principle should be constrained only by legal issues such as copyright - not by ethical norms and/or constraints that violate this humanistic principle.

Some argue that self-plagiarism represents ethical misconduct: for example, duplicated data can affect meta-analyses and waste precious publication space. But selfplagiarism is currently not a legal issue; it does not meet the US Public Health Service research misconduct standards.

As editor of an international journal, The Journal of Cognitive and Behavioral Psychotherapies, I believe that a comprehensive ban on self-plagiarism is a fundamental error.

The reader assumes, unless told otherwise, that the text is written by the author, and that it is novel and accurate. I believe that ethical writing in relation to self-plagiarism should be defined by: full disclosure if the new and/or derivative work incorporates text previously published; citing the old work in the new; and ensuring that there is no violation of copyright law.

If duplication of content within these constraints helps the author to reach a new or larger readership, and/or if text recycling within these constraints helps to present the same idea more accurately across several publications, they become legitimate conduct. Efforts to suppress the dissemination of scientific knowledge by overregulation call to mind the Inquisition, which was established to prevent spiritual wrong-doing in the Middle Ages.

Daniel David

Babes-Bolyai University,

No. 37 Republicii St, 400015,

Cluj-Napoca, Romania

\section{Duplication and plagiarism increasing among students}

SIR - Mounir Errami and Harold Garner make the point in their Commentary (Nature 451, 397-399; 2008) that plagiarism in scientific writing is on the increase. Ready access to electronic copy makes it easy to snip out that handy phrase that encapsulates a thought, or that neat introductory paragraph, and then why not the whole section, and so on. We have all been tempted.

Undergraduates regularly expropriate whole articles from Wikipedia. They are not scared by anti-plagiarism software, as they know it is not routinely applied. As today's undergraduates will become tomorrow's researchers, the problem can only get worse.

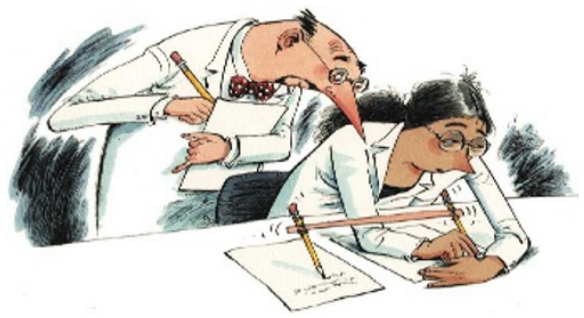

As a referee who is used to reviewing papers in particular niche areas, I have occasionally identified duplicate or seriously overlapping content in manuscripts, often intended for high-profile journals. In one instance, I received almost identical papers for review and in another I received a duplicate of an online pre-publication paper. The authors cannot be named, as that would violate referee confidentiality, but they were from well-known institutions in the developed world.

What these duplications had in common (apart from the text!) was that the authors were relatively junior and newly appointed. Younger academics seem to feel under a great deal of pressure to publish. In my own institution, I believe that my younger colleagues place an unhealthy emphasis on impact factor when considering where to publish their work.

How to prevent plagiarism? Journal editors must refuse plagiaristic pieces and explain why. Identification of duplication post-publication should lead to high-profile withdrawal of the papers by the journal that was misused. Plagiarism should be identified as a disciplinary offence in the employment contract of academic and research staff.

Brian Derby

School of Materials, University of Manchester, Grosvenor Street, Manchester M1 7HS, UK

\section{Duplication: most cases on database are innocent}

SIR - In a super-egotistical response to the Commentary about duplication (Nature 451, 397-399; 2008), I decided to check the Déjà vu database (http://spore.swmed.edu/dejavu) to see whether anybody was copying my data. A search for "Brennan, P." revealed 31 citations, but this came as no surprise, because my name is quite common. However, I was more surprised when I went through the list and found only two significant incidents of plagiarism. This indicates a $93 \%$ false-positive rate for genuine duplication on my small sample. The Commentary authors themselves report a false-positive rate of $27 \%$, after manually checking a much larger sample of 2,600 entries.

Some false positives could be excluded with a little effort. Four of the 31 articles were a series - more of a rarity today, but quite common in the past; sometimes the word 'part' appears in the title, so these could be excluded. Seven of the 31 were clinical updates and four were updates of reviews or opinion pieces. However, reviews and clinical trials are indicated in Medline and could probably be excluded from analysis. Clinical updates are often three or more years apart but have similar authors; the Déjà vu database has a 'time lag' field that can help to identify this type of duplication.

Errami and Garner ask whether it is reasonable to publish the same information in different languages, enabling a piece of work to reach different audiences. In terms of promoting good practice in medicine or other fields, local languages are important. These could be excluded within Medline.

Of the remaining articles I uncovered, there were one-off explanations: research on similar and related topics that seemed like duplicates but were, to me, distinct; re-publication of an article with corrections; and one example of an administrative error causing the journal to publish the article twice. One reprint was an homage to classic citation with permission. The Déjà vu database's tagging system is too simple to differentiate all of these contributions.

Paul Brennan

Medical Biochemistry and Immunology, School of Medicine, Cardiff University, Heath Park, Cardiff CF14 4XN, UK

Join in the duplication debate at http:// tinyurl.com/2koto4. 